\title{
Analysis of stress granule assembly in Schizosaccharomyces pombe
}

\author{
CHUN-YU WANG, ${ }^{1}$ WEI-LING WEN, ${ }^{1}$ DANIEL NILSSON, ${ }^{2}$ PER SUNNERHAGEN, ${ }^{2}$ TIEN-HSIEN CHANG, ${ }^{3}$ \\ and SHAO-WIN WANG ${ }^{1,4}$ \\ ${ }^{1}$ Institute of Molecular and Genomic Medicine, National Health Research Institutes, Zhunan Town, Miaoli County 350, Taiwan \\ ${ }^{2}$ Department of Cell and Molecular Biology, Lundberg Laboratory, University of Gothenburg, S-405 30 Göteborg, Sweden \\ ${ }^{3}$ Genomics Research Center, Academia Sinica, Nankang, Taipei 11529, Taiwan
}

\begin{abstract}
Stress granules (SGs) are cytoplasmic aggregates of RNA and proteins in eukaryotic cells that are rapidly induced in response to environmental stress, but are not seen in cells growing under favorable conditions. SGs have been primarily studied in mammalian cells. The existence of SGs in the fission yeast and the distantly related budding yeast was demonstrated only recently. In both species, they contain many orthologs of the proteins seen in mammalian SGs. In this study, we have characterized these proteins and determined their involvement in the assembly of fission yeast SGs, in particular, the homolog of human G3BP proteins. G3BP interacts with the deubiquitinating protease USP10 and plays an important role in the assembly of SGs. We have also identified Ubp3, an ortholog of USP10, as an interaction partner of the fission yeast G3BP-like protein Nxt3 and required for its stability. Under thermal stress, like their human orthologs, both Nxt3 and Ubp3 rapidly relocalize to cytoplasmic foci that contain the SG marker poly(A)-binding protein Pabp. However, in contrast to G3BP1 and USP10, neither deletion nor overexpression of $n x t 3^{+}$or $u b p 3^{+}$affected the assembly of fission yeast SGs as judged by the relocalization of Pabp. Similar results were observed in mutants defective in orthologs of SG components that are known to affect SG assembly in human and in budding yeast, such as ataxia-2 and TIA-like proteins. Together, our data indicate that despite similar protein compositions, the underlying molecular mechanisms for the assembly of SGs could be distinct between species.
\end{abstract}

Keywords: G3BP-like protein Nxt3; TIA-like proteins; ataxia two homolog Ath1; deubiquitinating protease Ubp3; fission yeast stress granules

\section{INTRODUCTION}

In response to environmental stress, eukaryotic cells shut down protein synthesis in a stereotypic response that conserves anabolic energy for the repair of stress-induced damage. This results in the disassembly of polyribosomes, leading to stalled initiation complexes that are dynamically recruited to cytoplasmic foci called stress granules (SGs) (Anderson and Kedersha 2009; Buchan and Parker 2009). SGs are not required for global translation repression or global mRNA stability. Instead, it has been proposed that SGs are sites where the increased local concentration of proteins and mRNA allows for remodeling and redistribution of messenger ribonucleoproteins (Buchan and Parker 2009). Alternatively, it has also been proposed that specific proteins can

\footnotetext{
${ }^{4}$ Corresponding author.

E-mail shaowinwang@nhri.org.tw.

Article published online ahead of print. Article and publication date are at http://www.rnajournal.org/cgi/doi/10.1261/rna.030270.111.
}

be selectively sequestered into or away from SGs, thus affecting biochemical processes in the cell. For example, recruitment of specific proteins such as RACK1, which is required to activate the apoptosis-inducing MTK1 kinase during stress, to SGs can inhibit apoptosis (Arimoto et al. 2008).

The primary trigger for SG assembly is the inhibition of protein synthesis, where stalled initiation complexes are shuttled to and aggregated into SG foci. SG formation can occur in cells that respond to environmental stresses or through the addition of chemicals that block the activity of specific translation initiation factors. One of the bestdescribed pathways is through eIF $2 \alpha$ phosphorylation (Kedersha et al. 1999, 2002). In response to distinct stresses, eIF $2 \alpha$ kinases are activated to target and phosphorylate eIF2 $\alpha$. This phosphorylation inhibits a key step in translation initiation, leading to stalling of initiation complexes on mRNA and their transfer to SGs (Kedersha et al. 1999). SGs can also be induced in an eIF2 $\alpha$-independent manner. For example, treatment of cells with hippistranol or pateamine A (PatA), which alters the activity of the helicase eIF4A, also induces 
SG formation (Dang et al. 2006; Mazroui et al. 2006). In general, inhibition of translation that results in the release of ribosomal subunits from translating complexes will trigger SG assembly. However, there are exceptions. In one study, preventing the $60 \mathrm{~S}$ subunit from joining with the $40 \mathrm{~S}$ subunit did not lead to SG assembly, suggesting that translational repression can be uncoupled from SG induction (Mokas et al. 2009). Thus, SGs may only form through the inhibition of specific translation factors or within a defined window during translation initiation.

SGs are cytoplasmic phase-dense particles composed of several proteins and RNA. Based on studies in mammalian cells, besides stalled initiation complexes composed of translation initiation factors such as eIF4E, eIF2, eIF3, PABP, and the small $40 \mathrm{~S}$ ribosomal subunit, SGs contain hallmark protein markers such as T-cell intracellular antigen-1 (TIA-1), TIA-1-related protein (TIAR), and Ras-GAP SH3 domain binding protein G3BP (Kedersha et al. 1999; Tourriere et al. 2003). TIA-1 and TIAR are closely related proteins that contain RNA recognition motifs and are implicated in RNA metabolism (Tian et al. 1991). G3BP, a member of the Ras signaling pathway, was discovered by its ability to bind to the SH3 domain of RasGAP and has since been implicated in several biological processes including RNA metabolism (Irvine et al. 2004). All three proteins contain domains important for the aggregation and formation of SGs. For example, TIA-1 and TIAR lacking the QN-rich prion-like domain, which promotes self-aggregation, can no longer form SGs (Gilks et al. 2004). Additional components required for the assembly of SGs include ataxin-2 (ATXN2), a polyglutamine (polyQ) protein mutated in spinocerebellar ataxia type 2, which interacts with the DEAD/H-box RNA helicase DDX6, a component of processing bodies (P-bodies), and colocalized in SGs in mammalian cells (Nonhoff et al. 2007).

SGs have been primarily studied in mammalian cells. The existence of SGs in the fission yeast Schizosaccharomyces pombe (Wen et al. 2010; Nilsson and Sunnerhagen 2011) and the distantly related budding yeast Saccharomyces cerevisiae (Hoyle et al. 2007; Buchan et al. 2008; Grousl et al. 2009) was demonstrated only recently. They contain many proteins analogous to those in mammalian SGs. Despite the fact that yeast SGs seem to contain most if not all components of mammalian SGs, unlike the situation in mammals, their formation is independent of eIF2 $\alpha$ phosphorylation in the budding yeast (Grousl et al. 2009). Similarly, observations from fission yeast (Wen et al. 2010; Nilsson and Sunnerhagen 2011) and trypanosomes (Kramer et al. 2008) show that assemblies of SGs in response to heat shock are also independent of phosphorylation of eIF $2 \alpha$. It appears that other pathways contribute to formation of SGs in several organisms. In keeping with this notion, here we describe the characterization of fission yeast SGs and have identified the ubiquitin protease Ubp3-Nxt3 complex as a novel component. We found that although the protein composition of SGs is similar between species, the requirements for their assembly are not. It will be intriguing in the future to investigate what differentiates fission yeast cells from other cell types in the assembly of SGs, and what are the underlying molecular mechanisms.

\section{RESULTS}

SGs are cytoplasmic aggregates that are not seen in eukaryotic cells growing under favorable conditions but are rapidly induced in response to environmental stress. Despite the efforts of several groups, no one has yet succeeded in purifying SGs, so their contents are morphologically defined using immunostaining and GFP-tagging of individual proteins (Kedersha and Anderson 2007; data not shown). To gain more insight into the protein composition of fission yeast SGs, in this study, we determined its components by generating chromosomally tagged GFP-fusion proteins of known mammalian SG components (Table 1) based on their localization under thermal stress with the SG marker Pabp tagged with mCherry. Microscopic screening of these strains detected additional SG markers as well as novel components including the deubiquitinating enzyme Ubp3 and its cofactor Nxt3 (G3BP homolog), Ath1 (ataxiatwo homolog), and Cxr1 (도시-related TIA-1 homolog). The dependencies of the assembly of fission yeast SGs on these proteins were also determined.

\section{Nxt3 as a component of fission yeast stress granules}

In human, G3BPs form a small family of three ubiquitously expressed proteins, derived from two distinct genes. In addition to G3BP1, the second member, G3BP2, exists in two differentially spliced forms (Irvine et al. 2004). The $\mathrm{N}$ terminus of the G3BPs is characterized by the presence of an NTF-2 (nuclear transport factor 2)-like domain and a segment rich in acidic residues (Fig. 1A). The central segment contains proline-rich PXXP motifs, and the C-terminal portion of the proteins is characterized by motifs associated

TABLE 1. List of fission yeast SG components in this study

\begin{tabular}{|c|c|c|}
\hline$S p$ & Sc & $H_{s}$ \\
\hline $\mathrm{Ubp}^{\mathrm{a}}$ & Ubp $3^{\mathrm{b}}$ & Usp10 c \\
\hline $\mathrm{Nxt} 3^{\mathrm{a}}$ & Bre $^{\text {b }}$ & $G 3 B P^{d}$ \\
\hline $\operatorname{Cs} x 1^{e}$ & Pub1 $^{f}$ & TIA-1 ${ }^{\mathrm{g}}$ \\
\hline $\mathrm{Cxr}^{\mathrm{a}}$ & $\operatorname{Ngr}^{f}$ & TIA-R ${ }^{g}$ \\
\hline Ath $1^{\mathrm{a}}$ & $\mathrm{Pbp}^{\mathrm{f}}$ & ATXN2 $2^{h}$ \\
\hline
\end{tabular}

${ }^{a}$ Novel component identified in this study.

${ }^{\mathrm{b}}$ Not determined.

${ }^{\mathrm{c}} \mathrm{Ohn}$ et al. (2008).

${ }^{\mathrm{d}}$ Tourriere et al. (2003).

eNilsson and Sunnerhagen (2011); role in SGs not determined.

fBuchan et al. (2008).

${ }^{g}$ Kedersha et al. (1999).

${ }^{\mathrm{h}}$ Nonhoff et al. (2007). 
A

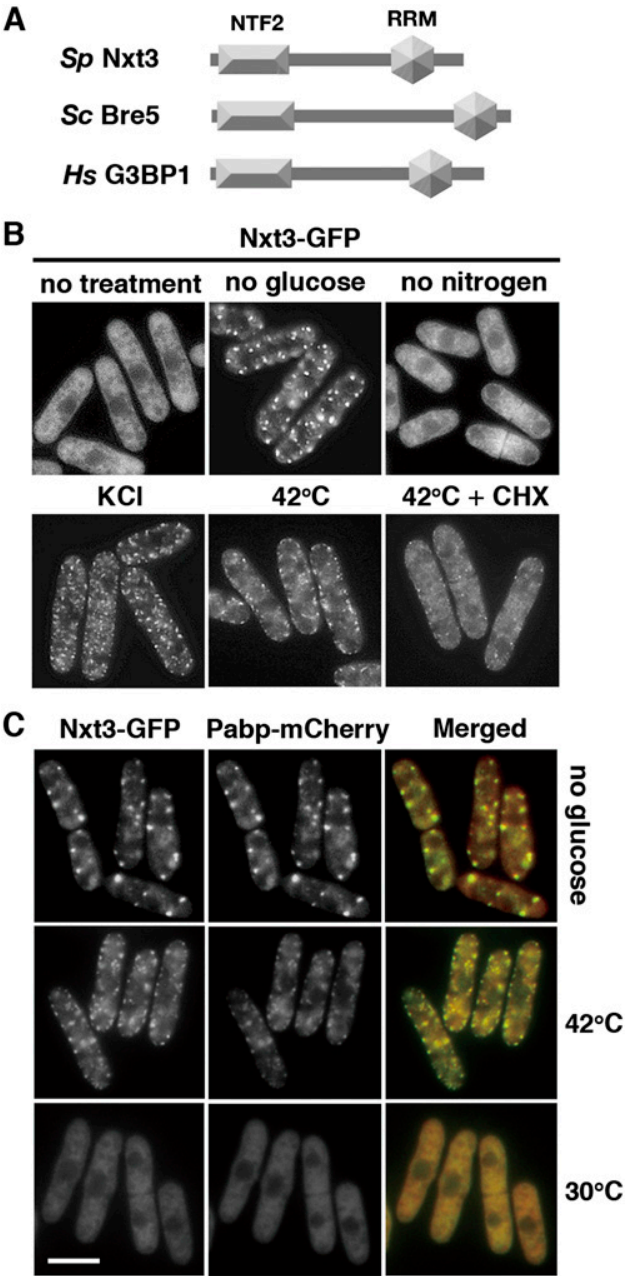

FIGURE 1. G3BP-like protein Nxt3 as a component of fission yeast SGs. (A) Schematic representation of the domain structures of S. pombe Nxt3, S. cerevisiae Bre5, and human G3BP1. (NTF2) Nuclear transport factor 2-like domain; (RRM) RNA recognition motif. $(B)$ Nxt3-GFP was visualized after growth to mid-logarithmic phase at $30^{\circ} \mathrm{C}$ (no treatment), or after deprivation of glucose or nitrogen for 1 and $3 \mathrm{~h}$, respectively, or after exposure to $1 \mathrm{M} \mathrm{KCl}$ and after a shift to $42^{\circ} \mathrm{C}$ for $15 \mathrm{~min}$. One hundred micrograms per milliliter $(100 \mu \mathrm{g} / \mathrm{mL})$ $\mathrm{CHX}$ was added $1 \mathrm{~min}$ before the shift to $42^{\circ} \mathrm{C}$. (C) Merged images of fluorescence micrographs showing Pabp-mCherry (red) and Nxt3GFP (green) localization in living cells grown at $30^{\circ} \mathrm{C}$, and after a 15 -min incubation at $42^{\circ} \mathrm{C}$, or deprivation of glucose for $1 \mathrm{~h}$. (Bar) $5 \mu \mathrm{m}$.

with RNA binding, including a canonical RNA recognition motif and an arginine- and glycine-rich RGG box. As in S. cerevisiae, S. pombe contains a single copy of the gene encoding the G3BP-like protein, Nxt3. To characterize Nxt3 in $S$. pombe, targeted recombination was used to add various epitope-tag sequences to the $3^{\prime}$ end of the $n x t 3^{+}$open reading frame in its normal chromosomal context. The tagged proteins appeared to be functional as judged by the lack of hypersensitivity to paromomycin (Palmer et al. 1979) of the $n x t 3-G F P$ and $n x t 3-T A P$ strains compared with the $n x t 3$ mutant (Supplemental Fig. 2B). Examination of living cells by fluorescence microscopy revealed a diffuse cytoplas- mic localization of the Nxt3-GFP fusion protein (Fig. 1B). To determine the role of Nxt3 in fission yeast SGs, we next examined the localization of Nxt3-GFP under various types of stresses. As shown in Figure 1B, nitrogen deprivation did not change the localization of Nxt3-GFP. In contrast, glucose starvation caused a rapid relocalization of Nxt3-GFP to distinct cytoplasmic granule-like structures. Similarly, after exposure of cells to $1 \mathrm{M} \mathrm{KCl}$ and under thermal stress, a rapid relocalization of Nxt3-GFP was observed, although the granules were less conspicuous than under glucose starvation. In addition, we observed that poly(A)-binding protein Pabp-mCherry colocalized almost completely with GFP fusions of Nxt3 under thermal stress and glucose starvation (Fig. 1C). This colocalization indicates that these proteins are components of a single granule with a composition similar to mammalian SGs. This is further supported by the fact that, as in mammalian cells, drugs such as cycloheximide (CHX), which stabilizes polyribosomes by freezing ribosomes on translating mRNA, inhibited the assembly of these granules (Fig. $1 \mathrm{~B}, 42^{\circ} \mathrm{C}+\mathrm{CHX}$ ). Together, these observations demonstrated that Nxt3, as its human ortholog G3BP, is a component of fission yeast SGs.

\section{Ubp3 as an Nxt3-interacting protein and a component of fission yeast SGs}

To gain more insight into its biological function, tandem affinity purification was performed to identify proteins associated with Nxt3. The two-step immunoglobulin G (IgG)Sepharose and calmodulin resin affinity steps were performed as described previously (Rigaut et al. 1999) on 10-L cultures of the Nxt3-TAP strain. The protein composition of the TAP complex was examined directly by silver staining. As shown in Figure 2B, multiple polypeptides were detected, and their identities were determined by MALDI MS/MS analysis. Ubp3, the ortholog of USP10 (Fig. 2A), was identified in the TAP complex. In addition, five ribosomal proteins (L3, L4, L13, S1, and S9) that copurified with Nxt3 were identified (Fig. 2B). The interaction between Nxt3 and Ubp3 was further confirmed by a reciprocal TAP experiment using Ubp3 as a bait (Fig. 2C) and coimmunoprecipitation (data not shown). In agreement with these results, the interaction between Nxt3 and Ubp3 was also identified in a recently described systematic proteomic analysis of the 20 S. pombe deubiquitinating enzymes (Kouranti et al. 2010). Given the interaction between Nxt3 and Ubp3, we next asked whether or not Ubp3, like its binding partner, is a component of the fission yeast SGs. To this end, targeted recombination was used to add tdTomato epitope-tag sequences to the $3^{\prime}$ end of the $u b p 3^{+}$open reading frame in its normal chromosomal context. The tagged protein appeared to be functional as judged by the lack of hypersensitivity to paromomycin of the ubp3-tdTomato strains compared with the $u b p 3$ mutant (Supplemental Fig. 2B). Examination of living cells by fluorescence microscopy re- 
A

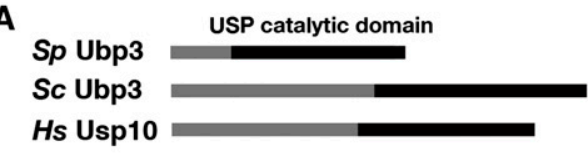

B

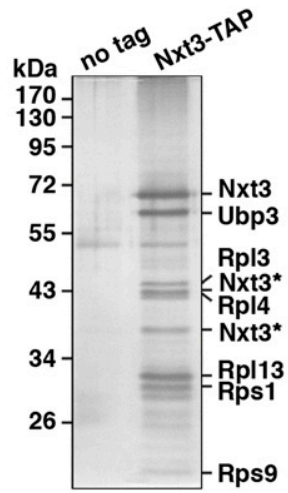

C

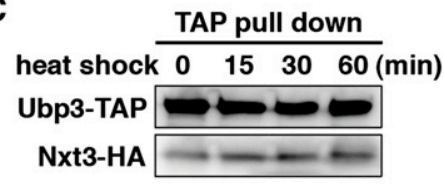

D Nxt3-GFP Ubp3-tdTomato Merged

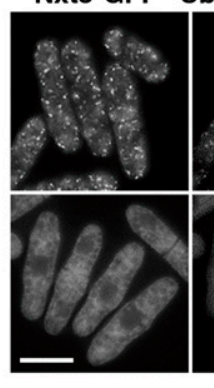

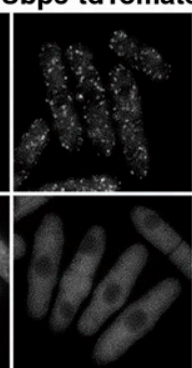

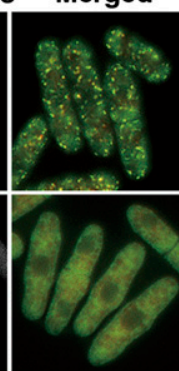

FIGURE 2. Ubp3 as an interaction protein of Nxt3. (A) Schematic representation of the domain structures of S. pombe Ubp3, S. cerevisiae Ubp3, and human Usp10. (B) Tandem affinity purification (TAP)-tag pull-down of Nxt3 proteins resolved by SDS-PAGE were visualized by silver staining. (Right) Identity of constituent proteins (identified by MALDI MS/MS analysis of individual bands). $\left(^{*}\right)$ Degradation products of Nxt3. (C) TAP-tag pulldown of Ubp3 proteins from strain expressing Nxt3-HA fusion proteins incubated at $42^{\circ} \mathrm{C}$ for the time indicated were separated by SDS-PAGE and subjected to Western blotting using antiPAP and anti-HA antibodies. (D) Merged images of fluorescence micrographs showing Ubp3tdTomato (red) and Nxt3-GFP (green) localization in living cells grown at $30^{\circ} \mathrm{C}$ and after a 15 -min incubation at $42^{\circ} \mathrm{C}$. (Bar) $5 \mu \mathrm{m}$.

veals a diffuse cytoplasmic localization of the Ubp3-tdTomato fusion protein. As shown in Figure 2D, thermal stress caused a rapid relocalization of these tdTomato-fusion proteins to distinct cytoplasmic granule-like structures that colocalized almost completely with the Nxt3-GFP protein. Together, these observations demonstrated that both Ubp3 and Nxt3 are components of fission yeast SGs.

\section{Assembly of Nxt3 and Ubp3 into SGs are independent of each other}

Next we probed the requirement of a complex formation between Nxt3 and Ubp3 for their assembly into SGs. To this end, strains lacking one protein and individually containing a GFP version of the other protein were constructed. Each strain was then examined microscopically for the accumulation of each protein in SGs under thermal stress. Intriguingly, fluorescent microscopy reveals a significant reduction of Nxt3 and Ubp3 protein levels in strains lacking the other protein (Fig. 3B), which was further confirmed by Western blot analysis (Fig. 3A), indicating that complex formation might help to stabilize the individual proteins. However, despite this strong interaction, Nxt3 and
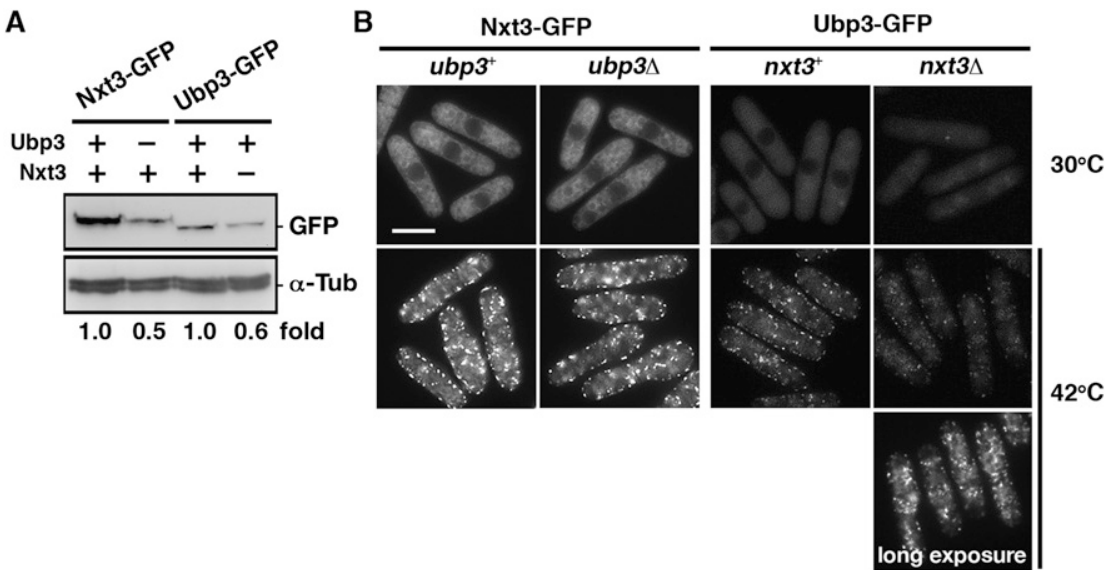

FIGURE 3. Assembly of Nxt3 and Ubp3 into SGs does not require their forming a complex with each other. (A) The Ubp3-GFP protein level was decreased in the $n x t 3$ mutant, and vice versa. Whole-cell protein extracts from the indicated strains expressing Nxt3 or Ubp3 GFPfusion proteins were separated by SDS-PAGE and subjected to Western blotting using antiGFP and anti-tubulin (loading control) antibodies. Relative intensities of the indicated proteins were indicated beneath each lane. $(B)$ Fluorescence micrographs of the indicated mutant strains expressing Nxt3 or Ubp3 GFP-fusion proteins grown at $30^{\circ} \mathrm{C}$ and after a 15 -min incubation at $42^{\circ} \mathrm{C}$. (Bar) $5 \mu \mathrm{m}$. $42^{\circ} \mathrm{C}$

$30^{\circ} \mathrm{C}$
Ubp3 granules could still form in the strain deleted for the interaction partner (Fig. 3B), suggesting that the assembly of Nxt3 and Ubp3 into SGs does not require complex formation between each other.

\section{Nxt3 and Ubp3 are dispensable for the formation of fission yeast SGs}

Both Usp10 and G3BP1 have been implicated in the formation of SGs (Tourriere et al. 2003; Ohn et al. 2008). To determine the role of the Ubp3-Nxt3 complex in fission yeast SG assembly, we examined the ability of $n x t 3$ and $u b p 3$ mutants to form SGs. As shown in Figure 4, A and B, we observed that SGs could still form in mutants lacking Nxt3, Ubp3, or both proteins, suggesting that, unlike their human orthologs, Nxt3 and Ubp 3 are not absolutely required for the assembly of fission yeast SGs, nor for their dissociation (Fig. 4C). In mammalian cells, SG assembly can be induced by G3BP overexpression. Cells transfected with a construct overexpressing G3BP-GFP concentrated the protein in aggregates corresponding to bona fide SGs (Tourriere et al. 2003). To test whether this might be applied to Nxt3, PCR-mediated gene disruption was normal chromosomal content with the thiamine-repress- 
A

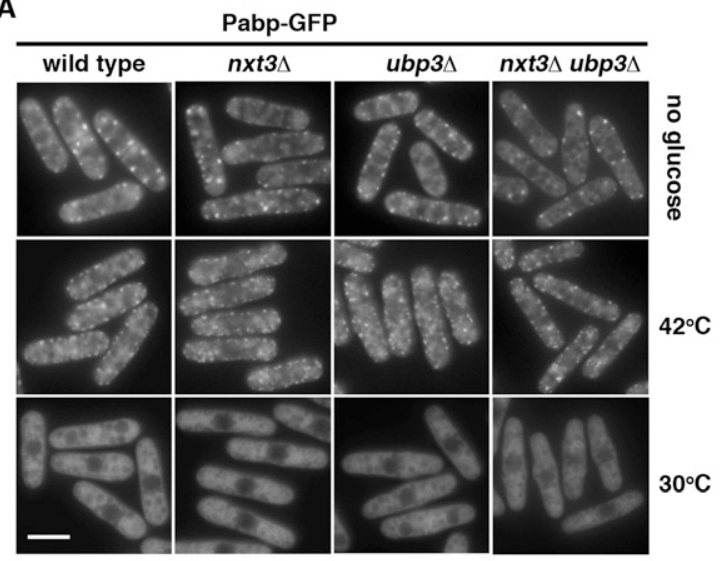

B

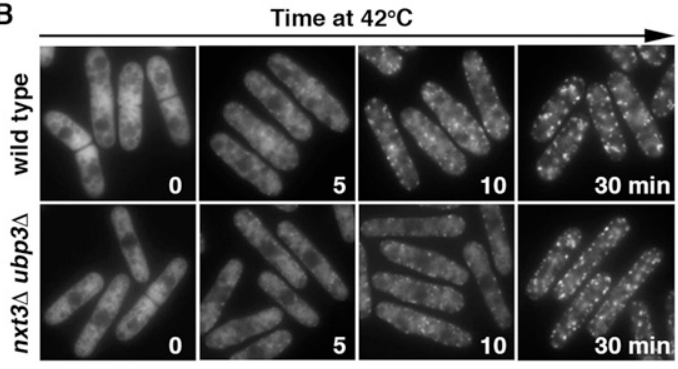

D

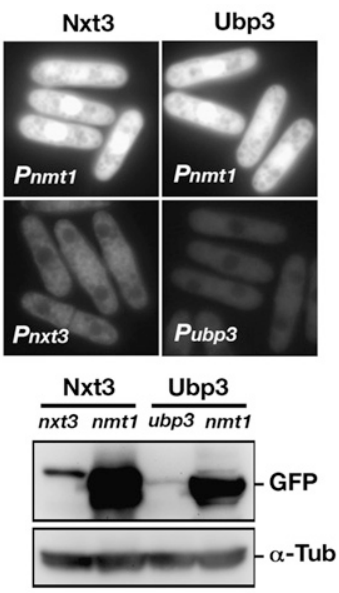

C Recovery

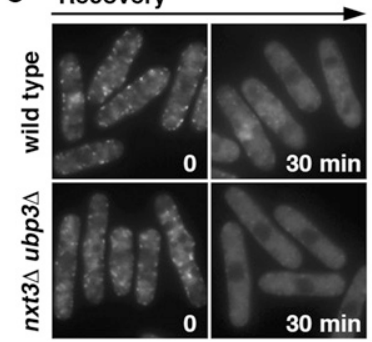

FIGURE 4. Nxt3 and Ubp3 are dispensable for the formation of fission yeast SGs. $(A)$ Fluorescence micrographs of the indicated strains expressing Pabp-GFP protein grown at $30^{\circ} \mathrm{C}$ and after a 15 -min incubation at $42^{\circ} \mathrm{C}$, or deprivation of glucose for $1 \mathrm{~h}$. (Bar) $5 \mu \mathrm{m}$. (B) PabpGFP was visualized in strains grown at $30^{\circ} \mathrm{C}$ (time 0 ) or after a shift to $42^{\circ} \mathrm{C}$ for the time indicated. $(C)$ Localization of Pabp-GFP after a 15 -min incubation at $42^{\circ} \mathrm{C}$ (time 0 ) followed by a shift back to $30^{\circ} \mathrm{C}$ for $30 \mathrm{~min}$. (D) Localization of the Nxt3 and Ubp3 GFP-fusion proteins under the control of its own promoter (Pnxt3 and Pubp3) or overexpressed by the nmt1 promoter (Pnmt1). (E) Whole-cell protein extracts from cells expressing Nxt3 or Ubp3 GFPfusion proteins under the control of its own promoter (Pnxt3 and Pubp3) or overexpressed by the nmt1 promoter (Pnmt1) were separated by SDS-PAGE and subjected to immunoblotting using anti-GFP antibodies to reveal the GFP-fusion proteins. Antibodies against $\alpha$-tubulin were used as controls.

ible nmt1 promoter, at the same time introducing a GFP epitope-tag sequence fused in-frame to the $5^{\prime}$ end of the $n x t 3^{+}$open reading frame. Removal of thiamine from the medium led to readily detectable levels of GFP-Nxt3 after $18 \mathrm{~h}$ of growth at $30^{\circ} \mathrm{C}$ (Fig. $4 \mathrm{E}$ ). Comparison with levels of Nxt3 expressed from the $n x t 3^{+}$promoter in its normal chromosomal content indicated that the $n m t 1$ promoterdriven gene was overexpressed at least 10 -fold in comparison with endogenous $n x t 3^{+}$. Even at this level of $n x t 3^{+}$ overexpression, however, no aggregates of protein were observed in these cells (Fig. 4D). Similar results were observed when Ubp3 was overexpressed. Together, we conclude that both Nxt3 and Ubp3 are dispensable for the formation of fission yeast SGs.

\section{ATXN2 homolog and TIA-like proteins are not required for the assembly of fission yeast SGs}

In addition to G3BP-like proteins, TIA-like proteins and ATXN2 homolog are known to affect the assembly of SGs in human (Gilks et al. 2004; Nonhoff et al. 2007) and in the distantly related budding yeast (Buchan et al. 2008; Grousl et al. 2009). To determine whether or not these proteins play a role in the assembly of fission yeast SGs, experiments were conducted to characterize their fission yeast orthologs. BLAST searches of the complete $S$. pombe genome using the human TIA-1 amino acid sequence as a query identified a similar $S$. pombe gene, $S P B C 23 E 6.01 c$, in addition to the already-identified TIA-like protein Csxl (Rodriguez-Gabriel et al. 2003). ProfileScan analysis of the 473-amino-acid sequence revealed multiple RNA recognition motif (RRM) domains over its entire length as in other TIA-like proteins (Fig. 5A). In recognition of this level of sequence conservation, and in deference of the existing gene name in $S$. pombe, we refer to this $S$. pombe gene as $\operatorname{cxrl}^{+}$(for $\underline{\operatorname{cs} x} 1$-related protein 1). In addition, an S. pombe gene, SPBC21B10.03c, was identified as the homolog of ataxin-2 (Fig. 6A; Albrecht et al. 2004). We refer to this $S$. pombe gene as ath $1^{+}$(for ataxiatwo homolog 1). To determine whether or not these proteins, like their human orthologs, are components of SGs, targeted recombination was used to generate GFP-fusion versions of the relevant proteins in their normal chromosomal context under the control of the endogenous promoter, expressing the full-length protein C-terminally tagged with GFP. As shown in Figures 5C and $6 \mathrm{~B}$, fluorescence microscopy reveals a diffuse cytoplasmic localization of Csx1, Cxr1, and Ath1-GFP fusion protein in cells growing under favorable conditions. Thermal stress and glucose starvation caused a rapid relocalization of these GFPfusion proteins to distinct cytoplasmic granule-like structures that colocalized to a certain degree with Pabp-mCherry. Together, these observations demonstrated that these proteins, like their human orthologs, are components of fission yeast SGs.

To determine whether or not these proteins play a role in the formation of SGs, we examined the ability of ath $1, \operatorname{cs} x 1$, and/or cxr1 mutants to form SGs. As shown in Figures 5D and 6C, we observed that SGs could still form in all mutants analyzed. To test whether or not these proteins might have redundant function with Ubp3-Nxt3 in SG assembly, we generated multiple gene deletion mutants between ath1, $\operatorname{cs} x 1, c x r 1$, and $u b p 3 n x t 3$ mutants (Figs. 5D, 6C). However, we observed that SGs could still form in all mutants analyzed; furthermore, no synergism was found in double or 


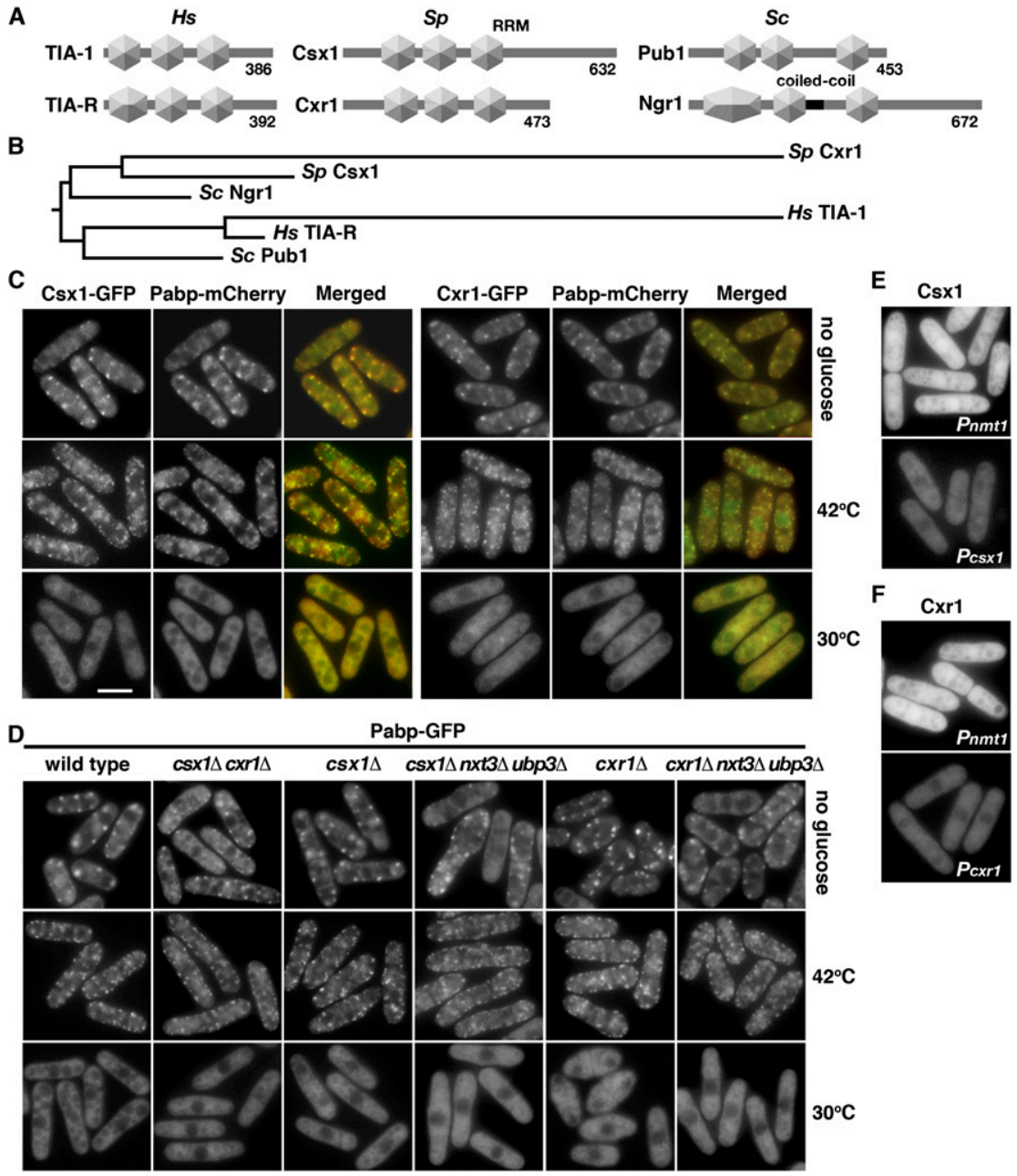

FIGURE 5. TIA-like proteins Csxl and Cxrl are not required for the assembly of fission yeast SGs. (A) Schematic representation of the domain structures of TIA-like proteins S. pombe Csx 1 and Cxr1, S. cerevisiae Pub1 and Ngr1, and human TIA-1 and TIA-R. (RRM) RNA recognition motif. (B) Cladogram showing the relationship between S. pombe Cxr1 and TIA-like proteins from S. cerevisiae to human. The length of each pair of branches represents the distance between sequence pairs. $(C)$ Merged images of fluorescence micrographs showing PabpmCherry (red) and TIA-like proteins' (Csxl and Cxr1; green) localization in living cells grown at $30^{\circ} \mathrm{C}$ and after a $15-\mathrm{min}$ incubation at $42^{\circ} \mathrm{C}$, or deprivation of glucose for $1 \mathrm{~h}$. (Bar) $5 \mu \mathrm{m}$. $(D)$ Fluorescence micrographs of the indicated mutant strains expressing Pabp-GFP protein grown at $30^{\circ} \mathrm{C}$ and after a 15 -min incubation at $42^{\circ} \mathrm{C}$, or deprivation of glucose for $1 \mathrm{~h}$. $(E)$ Localization of the Csx1 GFP-fusion protein under the control of its own promoter (Pcsxl) or overexpressed by the $n m t 1$ promoter $(P n m t 1)$. (F) Localization of the Cxrl GFP-fusion protein under the control of its own promoter $(P c x r 1)$ or overexpressed by the nmt1 promoter (Pnmt1).

triple mutants at least under thermal stress (Table 2). Similarly, no aggregates of these proteins were observed in cells when overexpressed (Figs. 5E,F, 6D). Together, we concluded that in fission yeast, TIA-like proteins and ATXN2 homolog Ath1 are dispensable for the formation of SGs. Although having a similar protein composition, the underlying molecular mechanisms for the assembly of SGs are distinct between $S$. pombe and other species.

Our data also reveal distinct features between components of fission yeast SGs. As shown in Supplemental Figure
$1 \mathrm{~A}$, not all mutants defective in components of SGs are hypersensitive to arsenite, a chemical used to induce SGs. In fact, $n x t 3$ mutants are more resistant to the treatment of arsenite and heat shock (data not shown), suggesting that these proteins might have different roles related to the function of SGs. As part of our analysis, we also asked whether or not these proteins might work through regulation of the translational activities of mRNAs functioning in stress protection by measuring protein levels of heat-shock proteins. However, the accumulation of heat-shock proteins under thermal stress still occurred in all mutants analyzed (Supplemental Fig. 1B).

\section{Ubp3-Nxt3 associates with polyribosome}

In addition to the interaction between Nxt3 and Ubp3, our data also revealed a close interaction between the Ubp3Nxt3 complex and ribosomal proteins (Fig. 2B). To further verify this association, we examined polyribosomes and ribosomal subunits for the presence of the Ubp3-Nxt3 complex. Cells containing Nxt3-TAP and Ubp3-GFP were grown at $30^{\circ} \mathrm{C}$ and harvested in the presence of $\mathrm{CHX}$. Cell extracts were fractionated on $7 \%-47 \%$ sucrose gradients, and immunoblots were used to compare the distribution of Nxt3 and Ubp3 with that of ribosomal protein L4 (Rpl401-GFP). Polyribosomes, as well as 40S, 60S, and $80 \mathrm{~S}$ ribosomal subunits were analyzed using absorbance at $260 \mathrm{~nm}$. As shown in Figure 7A, Nxt3 and Ubp3 were detected in $40 \mathrm{~S}, 60 \mathrm{~S}$, and $80 \mathrm{~S}$ particles as well as in fractions corresponding to polyribosomes. As expected, Rpl401 was found in fractions corresponding to $60 \mathrm{~S}$, $80 \mathrm{~S}$ subunits and polyribosomes. Together, these results revealed a close interaction between the Ubp3-Nxt3 complex and polyribosomes that might be required for its proper function. To investigate a potential function of the Ubp3-Nxt3 complex in translation, we measured the polyribosome profiles in the corresponding mutants. As shown in Figure 7B, no significant difference was observed in any mutant analyzed, suggesting that neither protein is a core component of polyribosomes required for their assembly. Next, we examined the sensitivity of $n x t 3$ and $u b p 3$ mutants to drugs that inhibit protein synthesis 
A

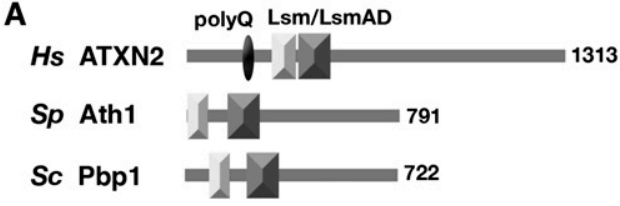

B

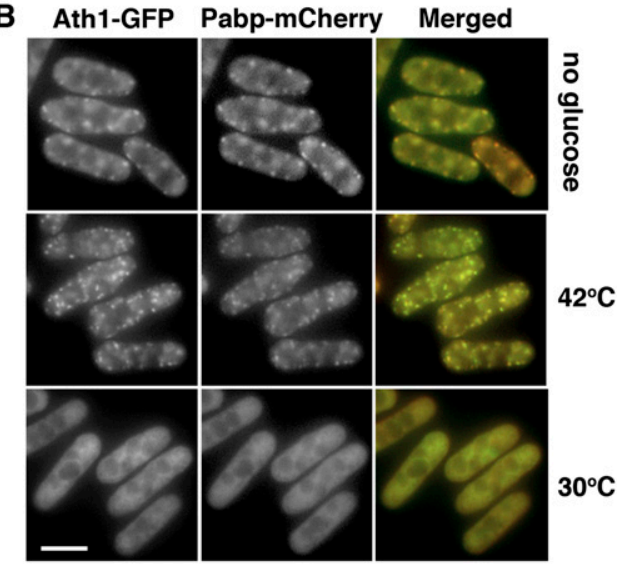

C

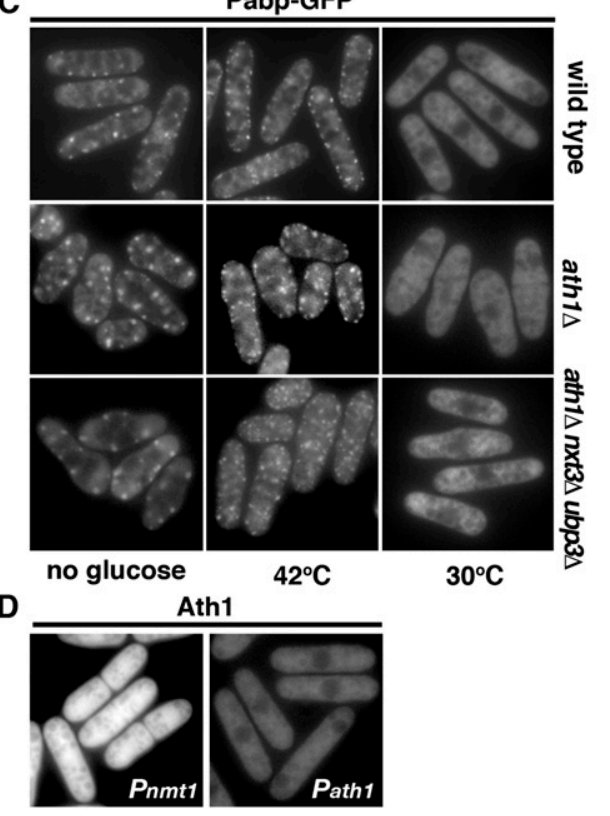

FIGURE 6. The ATXN2 homolog is not required for the assembly of fission yeast SGs. (A) Schematic representation of the domain structures of S. pombe Ath1, S. cerevisiae Pbp1, and human ATXN2. (B) Merged images of fluorescence micrographs showing PabpmCherry (red) and Ath1-GFP (green) localization in living cells grown at $30^{\circ} \mathrm{C}$ and after a 15 -min incubation at $42^{\circ} \mathrm{C}$, or deprivation of glucose for $1 \mathrm{~h}$. (Bar) $5 \mu \mathrm{m}$. (C) Fluorescence micrographs of the indicated mutant strains expressing Pabp-GFP protein grown at $30^{\circ} \mathrm{C}$ and after a $15-\mathrm{min}$ incubation at $42^{\circ} \mathrm{C}$, or deprivation of glucose for $1 \mathrm{~h}$. (D) Localization of the Ath1 GFP-fusion protein under the control of its own promoter (Path1) or overexpressed by the $n m t 1$ promoter (Pnmt1).

such as paromomycin, which increases codon misreading (Palmer et al. 1979), and CHX, which acts on the 60S subunit to inhibit peptide-bond formation. As shown in Supplemental Figure 2A, wild-type cells grew well on the YES medium containing $0.5 \mathrm{mg} / \mathrm{mL}$ paromomycin, whereas $n x t 3$ mutants, and to a lesser extent ubp3 mutants, failed to form colonies. No sensitivity was observed for mutants treated with CHX (data not shown). Similar results were observed for a strain defective in Vgl1, but not in Pabp (Supplemental Fig. 2A). Given that paromomycin did not induce SGs in these strains (data not shown), these results might reflect a function of these proteins in protein synthesis rather than a general role related to the function of SGs. Together, these observations revealed a close interaction between Ubp3-Nxt3 and polyribosomes that may be required for proper function of translation.

\section{DISCUSSION}

G3BPs were originally identified as displaying features of RNA-binding proteins and are found in various messenger ribonucleoprotein particles that regulate mRNA processing, transport, translation, and decay (Irvine et al. 2004). In particular, G3BP1 has been identified as a component of SGs (Tourriere et al. 2003). Not only is G3BP1 recruited for formation of SGs in response to cellular stresses, but SG assembly can also be induced by G3BP1 overexpression alone. In recent years, there has been a rapid expansion in our understanding of the role of SGs and G3BP1 in this response to cellular stress (Irvine et al. 2004). However, an understanding of the involvement of G3BP-like proteins in SG assembly in systems other than mammalian cells is still lacking. To this end, here we describe the characterization of a fission yeast G3BP-like protein, Nxt3, and have investigated its role in SG assembly. In addition, we have identified Ubp3, an ortholog of USP10, as an interacting protein partner of Nxt3 (Fig. 2). Intriguingly, in strains lacking either Nxt3 or Ubp3, we observed a significant reduction of the level of the respective protein partner

TABLE 2. The numbers of SGs in different genetic backgrounds measured after heat shock for $15 \mathrm{~min}$ at $42^{\circ} \mathrm{C}$

\begin{tabular}{|c|c|}
\hline Yeast strain & $\begin{array}{c}\text { Number of foci } \\
\text { per cell }\end{array}$ \\
\hline Wild type & $57 \pm 20$ \\
\hline$n x t 3 \Delta$ & $59 \pm 21$ \\
\hline ubp3s & $48 \pm 8$ \\
\hline$n x t 3 \Delta$ ubp3s & $53 \pm 19$ \\
\hline $\operatorname{cs} \times 1 \Delta \operatorname{cxr} 1 \Delta$ & $65 \pm 22$ \\
\hline $\operatorname{cs} \times 1 \Delta$ & $44 \pm 12$ \\
\hline $\operatorname{csx} 1 \Delta n x t 3 \Delta$ ubp3s & $79 \pm 22$ \\
\hline Cxr1 & $52 \pm 14$ \\
\hline cxr1s nxt3s ubp3s & $57 \pm 25$ \\
\hline ath1s & $52 \pm 14$ \\
\hline ath1 $n x t 3 \Delta$ ubp3s & $53 \pm 17$ \\
\hline
\end{tabular}

Quantified data sets involving Pabp-GFP markers represent the analysis of at least three independent experiments with a minimum of 50 cells scored for SG data. 
A

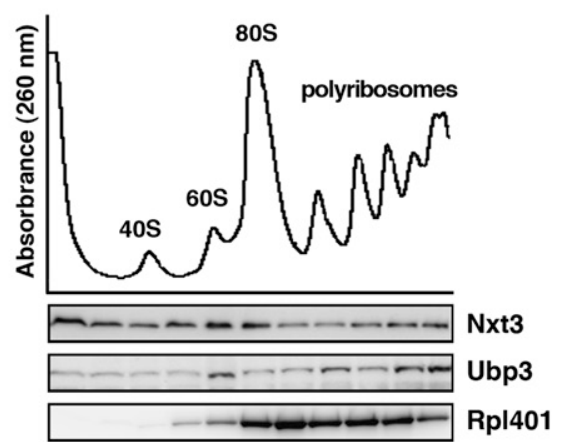

B

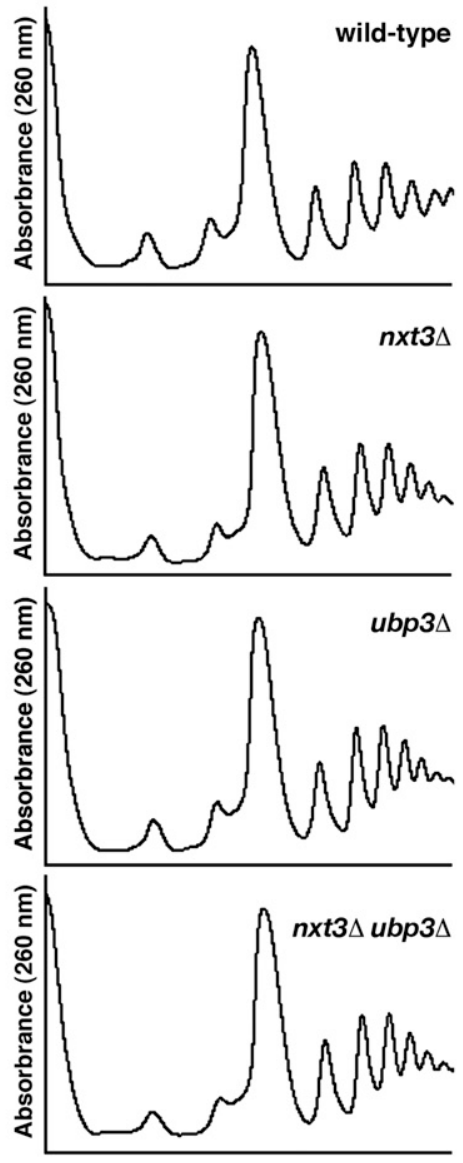

FIGURE 7. Ubp3-Nxt3 associates with polyribosome. (A) Cytosolic native extracts from $\mathrm{CHX}$-treated yeast cells grown at $30^{\circ} \mathrm{C}$ were separated on $7 \%-47 \%$ sucrose gradients. The distribution of the rRNA is shown by absorption profiles at $260 \mathrm{~nm}$ and the distribution of proteins of interest by Western blotting after denaturing gel electrophoresis. (B) Cytosolic native extracts from CHX-treated yeast strains as indicated were separated on $7 \%-47 \%$ sucrose gradients. The distribution of the rRNA is shown by absorption profiles at $260 \mathrm{~nm}$.

(Fig. 3), indicating that complex formation might help to stabilize the individual proteins. Our data also revealed a close interaction between the Ubp3-Nxt3 complex and ribosomal proteins (Fig. 2B). Furthermore, using sucrose gradient fractionation, we demonstrated that Nxt3 comigrates with polyribosomes (Fig. 7), as found in human neuronal cells for G3BP1 (Atlas et al. 2007), suggesting a function in translation. In line with these data, $n x t 3 \mathrm{mu}-$ tants are hypersensitive to drugs that inhibit protein synthesis such as paromomycin and displayed cold sensitivity, a characteristic feature of mutants with alteration in components of the translation apparatus (Supplemental Fig. 2; Shor et al. 2003).

Like its human orthologs, under thermal stress, Nxt3 was observed to rapidly relocalize to cytoplasmic foci that contain the SG marker poly(A)-binding protein Pabp. Like mammalian SGs, the assembly of these granules is blocked by CHX (Fig. 1B). Moreover, these granules contain many proteins analogous to those seen in mammalian SGs including Csx1, Cxr1, Ath1 (orthologs of the mammalian SG proteins TIA-1, TIAR, ATXN2, respectively) (Figs. 5, 6), and its binding partner Ubp3 (Fig. 2). However, despite the physical interaction between Nxt3 and Ubp3, and the requirement of the complex for protein stability, Nxt3 and Ubp3 granules could still form in strains lacking the other protein partner (Fig. 3), suggesting that the assembly of Nxt3 and Ubp3 into SGs does not require the reciprocal complex formation, and the individual proteins could be recruited to SGs through interaction with other proteins or mRNA. Although in S. cerevisiae Bre5 has been shown to positively regulate Ubp3-mediated biological function in vivo (Cohen et al. 2003), the genetic and cell-biological evidence presented in this study suggest a high degree of independent function between Ubp3 and Nxt3.

One important aspect of SG assembly is the proteinprotein interaction domain present on numerous RNAbinding proteins. For example, the G3BP1 protein has a dimerization domain that contributes to SG formation during arsenite stress (Tourriere et al. 2003). Moreover, several proteins involved in RNA metabolism contain QN-rich prion-like domains, and the ability of those domains to self-aggregate can promote SG assembly. For example, the RNA-binding proteins TIA-1 and TIA-R and their orthologs are found in SGs and contain a conserved QN-rich domain (Supplemental Fig. 3). However, to our surprise, neither Nxt3 nor TIA-like proteins (Csx1 and Cxr1) are required for the formation of fission yeast SGs (Figs. 4, 5). In keeping with these results, the QN-rich domain is not conserved among species (Supplemental Fig. 3). Similar results were observed in ath1 mutants, defective in the fission yeast homolog of ATXN2, a component of SGs that is known to affect its assembly in human and the distantly related budding yeast (Fig. 6). Experiments will be further extended to other factors such as components of P-bodies that are known to affect SGs in S. cerevisiae (Buchan et al. 2008). Together, these results indicate that although fission yeast SGs have similar protein compositions to those of the SGs found in other species, the requirements for their assembly appear to be distinct among various species.

At present, the underlying molecular mechanism for the assembly of fission yeast SGs is not clear. One possible mechanism is through protein modification. For example, 
in human, acetylation has been shown to affect SGs (Kwon et al. 2007). HDAC6 deacetylase mutants are impaired in SG formation, and although the target of this activity is unclear, two feasible candidates include Hsp90 and microtubules. In addition, SGs contain ubiquitin-modified proteins (Kwon et al. 2007), and mutations in the ubiquitin-binding domain of HDAC6 (Kwon et al. 2007) and knockdowns of several factors implicated in ubiquitin metabolism affect SG formation (Ohn et al. 2008). Modification of proteins with O-Glc-NAc also enhances SG formation (Ohn et al. 2008). However, the hexosamine biosynthetic enzymes are lacking in yeast. Finally, methylation, or the ability to bind methyl groups via the Tudor domain, is necessary for localization of specific SG components (De Leeuw et al. 2007; Goulet et al. 2008), or their ability to drive SG formation when overexpressed (Hua and Zhou 2004). Elucidating the key physiological targets of various modifications in fission yeast SGs and the mechanisms underlying their effects will therefore be our future goal, so that the $S$. pombe system can be used in the future to explore the functional consequences of perturbation of SGs.

\section{MATERIALS AND METHODS}

\section{Fission yeast strains and methods}

Conditions for growth, maintenance, and genetic manipulation of fission yeast were as described previously (Moreno et al. 1991). A complete list of the strains used in this study is given in Supplemental Table 1. One-step gene disruption or modification via homologous recombination was performed following PCR-mediated generation of $\mathrm{ura}^{+}{ }^{+}$or KanMX selectable cassettes flanked by 80 -bp segments from appropriate regions of the genes of interest using the oligonucleotides described in Supplemental Table 2. The original cs $x 1$ strains (Rodriguez-Gabriel et al. 2003) were gifts from Dr. P. Russell. Except where otherwise stated, strains were grown at $30^{\circ} \mathrm{C}$ in yeast extract (YE) or Edinburgh Minimal Medium (EMM2) with appropriate supplements. Where necessary, gene expression from the $n m t 1$ promoter was repressed by the addition of $5 \mu \mathrm{M}$ thiamine to the culture medium.

\section{Microscopy and image quantitation}

Visualization of mCherry- and green fluorescent protein (GFP)tagged proteins in living cells was performed at room temperature. Images were acquired using a Leica DM RA2 microscope equipped with a Leica DC 350F camera and were assembled using Adobe PhotoShop. For quantitation, images were acquired using a Leica TCS SP5 confocal microscope with Z-series compilations of 10 images per stack. Data from at least three independent experiments with a minimum of 50 cells were used for quantitation. Image analysis was carried out by Metamorph Software version 7.7 according to the manufacturer's instructions.

\section{Polyribosome profile analysis}

Polyribosomes were obtained as previously described (Wen et al. 2010). CHX (100 $\mu \mathrm{g} / \mathrm{mL}$ ) was added to $50 \mathrm{~mL}$ of cultures at an
$\mathrm{OD}_{600}$ of 0.5 grown at $30^{\circ} \mathrm{C}$. The cultures were harvested and processed for polyribosome profiling on $7 \%-47 \%$ sucrose gradients. Profiles were obtained by online measurements of $A_{260 \mathrm{~nm}}$. Proteins in the polyribosome profile fractions were concentrated by trichloroacetic acid (TCA) precipitation, and samples were analyzed by SDS-PAGE and immunoblotting.

\section{Antibodies and immunoblotting}

Whole-cell protein extracts were prepared by alkaline extraction as described previously (Wen et al. 2010) and separated by SDS-PAGE before being subjected to immunoblotting using anti-GFP (Abchem) and anti-PAP (Peroxidase-Anti-Peroxidase soluble complex; SigmaAldrich) antibody to reveal tandem affinity purification (TAP)tagged proteins. Anti-HSP70 polyclonal antibody (SPA-757), which cross-reacted with yeast proteins, was from Stressgen. Anti-HAperoxidase high-affinity (3F10) antibody was from Roche. Antibody against $\alpha$-tubulin (Sigma-Aldrich) was used as control.

\section{Tandem affinity purification and protein identification}

Ten liters of cell culture grown to $1.0 \times 10^{7}$ to $2.0 \times 10^{7}$ cells $/ \mathrm{mL}$ in YE medium was harvested by centrifugation at $4^{\circ} \mathrm{C}$. After one round of washing in cold water, the cells were homogenized in liquid nitrogen followed by resuspending in $100 \mathrm{~mL}$ of NP-40 buffer $\left(6 \mathrm{mM} \mathrm{Na}_{2} \mathrm{HPO}_{4}, 4 \mathrm{mM} \mathrm{NaH} \mathrm{PO}_{4}, 1 \% \mathrm{NP}-40,150 \mathrm{mM}\right.$ $\mathrm{NaCl}, 2 \mathrm{mM}$ EDTA, $50 \mathrm{mM} \mathrm{NaF}, 0.1 \mathrm{mM} \mathrm{Na}_{3} \mathrm{VO}_{4}, 1 \mathrm{mM}$ PMSF, $1 \mathrm{mM}$ DTT, Complete protease inhibitor cocktail [Roche]). Cell extracts were centrifuged at $100,000 \mathrm{~g}$ for $1 \mathrm{~h}$ at $4^{\circ} \mathrm{C}$. The supernatants were incubated with immunoglobulin G-Sepharose beads (GE Healthcare) for $2 \mathrm{~h}$ at $4^{\circ} \mathrm{C}$. Beads were washed in IPP150 buffer $(10 \mathrm{mM}$ Tris at $\mathrm{pH} 8,150 \mathrm{mM} \mathrm{NaCl}, 0.1 \% \mathrm{NP}-40)$ on columns and cleaved off with 100 units of TEV protease (Invitrogen) in TEVcleaving buffer $(10 \mathrm{mM}$ Tris- $\mathrm{HCl}$ at $\mathrm{pH} 8$, $150 \mathrm{mM} \mathrm{NaCl}, 0.1 \% \mathrm{NP}-40,1 \mathrm{mM}$ DTT, $0.5 \mathrm{mM}$ EDTA) for $16 \mathrm{~h}$ at $4^{\circ} \mathrm{C}$. After washing and eluting, $\mathrm{CaCl}_{2}$ was added to the eluted proteins to a final concentration of $2 \mathrm{mM}$, and the extracts were incubated with calmodulin resin for $3 \mathrm{~h}$ at $4^{\circ} \mathrm{C}$. The purified proteins were eluted with $20 \mathrm{mM}$ EGTA to be precipitated with TCA at a final concentration of $20 \%$, separated on SDS-PAGE gels, and visualized by Sypro Ruby. Protein bands were excised from the gels, treated with trypsin, and subjected to concerted MALDI peptide mass fingerprinting (PMF) and CID MS/MS analysis for protein identification using a dedicated Q-Tof Ultima MALDI instrument (Micromass) by the Core Facilities for Proteomics and Glycomics located at the Institute of Biological Chemistry, Academia Sinica. The instrument was operated under MassLynx 4.0, and raw MS data were processed for database searching using ProteinLynx Global Server 2.0 against the SWISSPROT database with the Mascot program.

\section{SUPPLEMENTAL MATERIAL}

Supplemental material is available for this article.

\section{ACKNOWLEDGMENTS}

This work was supported by the National Health Research Institute, Taiwan (MG-099-PP-10) and the Swedish Research Council 
(2010-4645). We thank Dr. Paul Russell for yeast strains. Proteomic mass spectrometry analyses were performed by the Core Facilities for Proteomics and Glycomics located at the Institute of Biological Chemistry, Academia Sinica, supported by a National Science Council grant (NSC 98-3112-P-001-023) and the Academia Sinica.

Received September 6, 2011; accepted December 11, 2011.

\section{REFERENCES}

Albrecht M, Golatta M, Wullner U, Lengauer T. 2004. Structural and functional analysis of ataxin-2 and ataxin-3. Eur J Biochem 271: 3155-3170.

Anderson P, Kedersha N. 2009. RNA granules: Post-transcriptional and epigenetic modulators of gene expression. Nat Rev Mol Cell Biol 10: 430-436.

Arimoto K, Fukuda H, Imajoh-Ohmi S, Saito H, Takekawa M. 2008. Formation of stress granules inhibits apoptosis by suppressing stress-responsive MAPK pathways. Nat Cell Biol 10: 1324-1332.

Atlas R, Behar L, Sapoznik S, Ginzburg I. 2007. Dynamic association with polysomes during P19 neuronal differentiation and an untranslated-region-dependent translation regulation of the tau mRNA by the tau mRNA-associated proteins IMP1, HuD, and G3BP1. J Neurosci Res 85: 173-183.

Buchan JR, Parker R. 2009. Eukaryotic stress granules: The ins and outs of translation. Mol Cell 36: 932-941.

Buchan JR, Muhlrad D, Parker R. 2008. P bodies promote stress granule assembly in Saccharomyces cerevisiae. J Cell Biol 183: 441-455.

Cohen M, Stutz F, Belgareh N, Haguenauer-Tsapis R, Dargemont C. 2003. Ubp3 requires a cofactor, Bre5, to specifically de-ubiquitinate the COPII protein, Sec23. Nat Cell Biol 5: 661-667.

Dang Y, Kedersha N, Low WK, Romo D, Gorospe M, Kaufman R, Anderson P, Liu JO. 2006. Eukaryotic initiation factor $2 \alpha$-independent pathway of stress granule induction by the natural product pateamine A. J Biol Chem 281: 32870-32878.

De Leeuw F, Zhang T, Wauquier C, Huez G, Kruys V, Gueydan C. 2007. The cold-inducible RNA-binding protein migrates from the nucleus to cytoplasmic stress granules by a methylation-dependent mechanism and acts as a translational repressor. Exp Cell Res 313: 4130-4144.

Gilks N, Kedersha N, Ayodele M, Shen L, Stoecklin G, Dember LM, Anderson P. 2004. Stress granule assembly is mediated by prionlike aggregation of TIA-1. Mol Biol Cell 15: 5383-5398.

Goulet I, Boisvenue S, Mokas S, Mazroui R, Cote J. 2008. TDRD3, a novel Tudor domain-containing protein, localizes to cytoplasmic stress granules. Hum Mol Genet 17: 3055-3074.

Grousl T, Ivanov P, Frydlova I, Vasicova P, Janda F, Vojtova J, Malinska K, Malcova I, Novakova L, Janoskova D, et al. 2009. Robust heat shock induces eIF2 $\alpha$-phosphorylation-independent assembly of stress granules containing eIF3 and 40S ribosomal subunits in budding yeast, Saccharomyces cerevisiae. J Cell Sci 122: 2078-2088.

Hoyle NP, Castelli LM, Campbell SG, Holmes LE, Ashe MP. 2007. Stress-dependent relocalization of translationally primed mRNPs to cytoplasmic granules that are kinetically and spatially distinct from P-bodies. J Cell Biol 179: 65-74.

Hua Y, Zhou J. 2004. Survival motor neuron protein facilitates assembly of stress granules. FEBS Lett 572: 69-74.

Irvine K, Stirling R, Hume D, Kennedy D. 2004. Rasputin, more promiscuous than ever: A review of G3BP. Int J Dev Biol 48: 10651077.

Kedersha N, Anderson P. 2007. Mammalian stress granules and processing bodies. Methods Enzymol 431: 61-81.

Kedersha NL, Gupta M, Li W, Miller I, Anderson P. 1999. RNAbinding proteins TIA-1 and TIAR link the phosphorylation of
eIF- $2 \alpha$ to the assembly of mammalian stress granules. J Cell Biol 147: 1431-1442.

Kedersha N, Chen S, Gilks N, Li W, Miller IJ, Stahl J, Anderson P. 2002. Evidence that ternary complex (eIF2-GTP-tRNA ${ }_{i}^{\text {Met }}$ )-deficient preinitiation complexes are core constituents of mammalian stress granules. Mol Biol Cell 13: 195-210.

Kouranti I, McLean JR, Feoktistova A, Liang P, Johnson AE, RobertsGalbraith RH, Gould KL. 2010. A global census of fission yeast deubiquitinating enzyme localization and interaction networks reveals distinct compartmentalization profiles and overlapping functions in endocytosis and polarity. PLoS Biol 8: e1000471. doi: 10.1371/ journal.pbio.1000471.

Kramer S, Queiroz R, Ellis L, Webb H, Hoheisel JD, Clayton C, Carrington M. 2008. Heat shock causes a decrease in polysomes and the appearance of stress granules in trypanosomes independently of eIF2 $\alpha$ phosphorylation at Thr169. J Cell Sci 121: 3002-3014.

Kwon S, Zhang Y, Matthias P. 2007. The deacetylase HDAC6 is a novel critical component of stress granules involved in the stress response. Genes Dev 21: 3381-3394.

Mazroui R, Sukarieh R, Bordeleau ME, Kaufman RJ, Northcote P, Tanaka J, Gallouzi I, Pelletier J. 2006. Inhibition of ribosome recruitment induces stress granule formation independently of eukaryotic initiation factor $2 \alpha$ phosphorylation. Mol Biol Cell 17: 4212-4219.

Mokas S, Mills JR, Garreau C, Fournier MJ, Robert F, Arya P, Kaufman RJ, Pelletier J, Mazroui R. 2009. Uncoupling stress granule assembly and translation initiation inhibition. Mol Biol Cell 20: 2673-2683.

Moreno S, Klar A, Nurse P. 1991. Molecular genetic analysis of fission yeast Schizosaccharomyces pombe. Methods Enzymol 194: 795-823.

Nilsson D, Sunnerhagen P. 2011. Cellular stress induces cytoplasmic RNA granules in fission yeast. RNA 17: 120-133.

Nonhoff U, Ralser M, Welzel F, Piccini I, Balzereit D, Yaspo ML, Lehrach H, Krobitsch S. 2007. Ataxin-2 interacts with the DEAD/ H-box RNA helicase DDX6 and interferes with P-bodies and stress granules. Mol Biol Cell 18: 1385-1396.

Ohn T, Kedersha N, Hickman T, Tisdale S, Anderson P. 2008. A functional RNAi screen links O-GlcNAc modification of ribosomal proteins to stress granule and processing body assembly. Nat Cell Biol 10: 1224-1231.

Palmer E, Wilhelm JM, Sherman F. 1979. Variation of phenotypic suppression due to the $\psi^{+}$and $\psi^{-}$extrachromosomal determinants in yeast. $J$ Mol Biol 128: 107-110.

Rigaut G, Shevchenko A, Rutz B, Wilm M, Mann M, Seraphin B. 1999. A generic protein purification method for protein complex characterization and proteome exploration. Nat Biotechnol 17: 1030-1032.

Rodriguez-Gabriel MA, Burns G, McDonald WH, Martin V, Yates JR III, Bahler J, Russell P. 2003. RNA-binding protein Csx1 mediates global control of gene expression in response to oxidative stress. EMBO J 22: 6256-6266.

Shor B, Calaycay J, Rushbrook J, McLeod M. 2003. Cpc2/RACK1 is a ribosome-associated protein that promotes efficient translation in Schizosaccharomyces pombe. J Biol Chem 278: 49119-49128.

Tian Q, Streuli M, Saito H, Schlossman SF, Anderson P. 1991. A polyadenylate binding protein localized to the granules of cytolytic lymphocytes induces DNA fragmentation in target cells. Cell 67: 629-639.

Tourriere H, Chebli K, Zekri L, Courselaud B, Blanchard JM, Bertrand E, Tazi J. 2003. The RasGAP-associated endoribonuclease G3BP assembles stress granules. J Cell Biol 160: 823-831.

Wen WL, Stevenson AL, Wang CY, Chen HJ, Kearsey SE, Norbury CJ, Watt S, Bahler J, Wang SW. 2010. Vgl1, a multi-KH domain protein, is a novel component of the fission yeast stress granules required for cell survival under thermal stress. Nucleic Acids Res 38: $6555-6566$. 

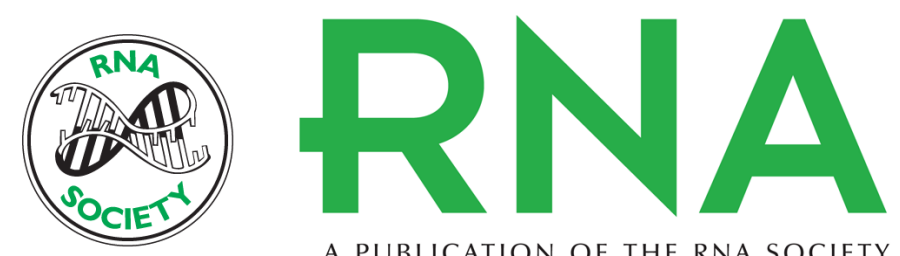

A PUBLICATION OF THE RNA SOCIETY

\section{Analysis of stress granule assembly in Schizosaccharomyces pombe}

Chun-Yu Wang, Wei-Ling Wen, Daniel Nilsson, et al.

RNA 2012 18: 694-703 originally published online February 10, 2012

Access the most recent version at doi:10.1261/rna.030270.111

Supplemental

Material

References

License

Email Alerting

Service
http://rnajournal.cshlp.org/content/suppl/2012/01/25/rna.030270.111.DC1

This article cites 34 articles, 16 of which can be accessed free at: http://rnajournal.cshlp.org/content/18/4/694.full.html\#ref-list-1

\section{top right corner of the article or click here.}

Receive free email alerts when new articles cite this article - sign up in the box at the

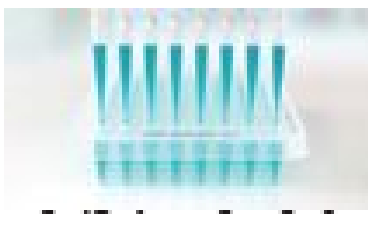

